\title{
Report on a conference on motor prostheses for workplace mobility of paraplegic patients in North America ${ }^{1}$
}

\author{
C Kantor, ${ }^{1} \mathrm{~B} \mathrm{~J}$ Andrews $\mathrm{PhD},{ }^{2} \mathrm{E}$ B Marsolais MD $\mathrm{PhD},{ }^{3 *} \mathrm{M}$ Solomonow $\mathrm{PhD},{ }^{4}$ \\ $\mathrm{R}$ D Lew MD, ${ }^{3} \mathrm{~K}$ T Ragnarsson $\mathrm{MD}^{5}$
}

${ }^{1}$ Tantalus Inc., Highland Park, NJ 08904, USA; ${ }^{2}$ University of Alberta, Edmonton, Canada; ${ }^{3} V A$ Medical Center, Cleveland, OH 44118, USA $;{ }^{4}$ LSU Medical Center, New Orleans, LA 70112, USA; ${ }^{5}$ Mt. Sinai Medical Center, New York, NY, USA.

\begin{abstract}
On May 18, 1992 a symposium at Case Western Reserve University in Cleveland, Ohio, USA had the goal of defining the tasks needed to reach clinical utility of investigational neural prosthetic ambulation devices. The characteristics and stage of development of four systems were detailed: the Lousiana State University reciprocating gait orthosis (LSU-RGO) with muscle stimulation; the modular hybrid functional neuromuscular stimulation (FNS) orthosis; the Cleveland VA-Case Western Reserve University (VA-CWRU) implant system; and the Parastep ${ }^{\circledR}$ system. Multicenter clinical trials are underway for the Parastep ${ }^{\circledR}$ system and are planned to start within the next 2 years for the LSU-RGO with muscle stimulation, the VA-CWRU 8-channel system, and the floor reaction orthosis component of the modular hybrid FNS system. Current investigational systems provide little advantage over the standing wheelchair in some occupations but they do expand social, recreational, and exercise capabilities. Disabled people and some leading rehabilitation physicians are willing to test basic ambulation devices but the regulatory approvals must first be obtained for multicenter clinical trials. Corporate partners are central to the development of devices, their clinical testing, and their subsequent marketing. A key requirement for developing and disseminating motor prostheses is the education of clinicians so that they will participate in trials and be prepared to prescribe the prostheses when they reach the market.
\end{abstract}

Keywords: paraplegia; functional neuromuscular stimulation (FNS): functional electrical stimulation (FES); motor prostheses; ambulation; functional restoration.

\section{Introduction}

While the wheelchair provides paralyzed people with energy-efficient transportation in many situations, there is a continuing interest in ambulation devices which can restore a more normal level of mobility. In addition to walking, that would include the ability to stand up, to climb and descend stairs, and to maneuver in small spaces. Orthoses have been available for many years, but they have often been rejected

*Correspondence: Dr E B Marsolais, Cleveland V A Medical Center, 10701 East Boulevard, Cleveland, Ohio 44106, USA. because they do not fully correct the functional loss, they are cumbersome, and they demand a large energy output from the user. In recent decades, advances in the ability to control the neuromuscular system with electrical stimulation have built a foundation for the development of neuroprosthetic devices for ambulatory mobility in paralyzed people.

Since the 1950s, physicians, engineers, therapists, and orthotists have designed and tested motor prosthetic devices which combine some degree of orthotic support with electrical stimulation. Despite significant progress, there is still no widely available clinical application of any of these systems. 
The fundamental design problems for providing ambulation for paraplegic patients have been summarized by Stallard et al. ${ }^{1}$ With the goal of defining the tasks needed to reach clinical utility, J Thomas Mortimer, director of the Applied Neural Control Laboratory of Case Western Reserve University in Cleveland, Ohio, USA, posed a focus question and provided a forum for the authors and discussants to present their perspectives on it. At the Seventh Annual Applied Neural Control Research Day on May 18, 1992, the participants responded to the question: What will it take to get 100 users of lower extremity neuroprostheses into the workplace? Carole Kantor, a medical writer, has compiled this paper from the proceedings of the symposium.

\section{Definitions and biomechanical requirements for motor prosthetic systems}

A neuroprosthetic user is an individual who regularly uses the device as an aid in activities of daily living. A workplace is a location in which a productive activity is performed: a home, an office, a school, or a factory.

The biomechanical principles of locomotion require both static and dynamic stability. Static stability can be provided by anti-gravity supports at all affected joints. Once the patient can stand and oppose gravity, dynamic stability requires balance to prevent a fall and to enable performance of useful functions. Locomotion in its simplest form can be performed with a stiff ankle and knee, allowing only one degree of freedom, flexion and extension. That can be accomplished if one leg undergoes hip flexion to yield the swing phase while the contralateral leg performs simultaneous hip extension and abduction to yield the pushoff and clearance of the swing leg, resulting in forward progression. Finally, one has to be able to independently stand up from the seated position or else independence is compromised.

A more cosmetic and natural form of walking requires that the knee, ankle, and hip be able to bend in normal ranges and that the spine be able to move. That necessitates more channels of electrical stimulation to control the joints and consequently less control from the mechanical components of the orthotic system. Any combination of electrical stimulation and orthoses must provide the user with a simple control interface in order that it be useful in the workplace. Since different occupations require different functional movements, each paralyzed worker will have individual requirements for leaning, reaching, and carrying objects. Using the system must be comfortably within the person's energy capacity.

For each of three neuroprosthetic ambulation systems we will describe the current system and the steps needed to deploy systems for practical daily use. First, Dr Moshe Solomonow will discuss the reciprocating gait orthosis (RGO) which has been developed at Louisiana State University (LSU) Medical Center in New Orleans, LA, USA. Second, Dr Brian J Andrews will review the modular hybrid functional neuromuscular stimulation (FNS) orthosis originating from his work at the University of Strathclyde in Glasgow, Scotland and now being further developed at the University of Alberta in Edmonton, Alberta, Canada. Third, Dr E Byron Marsolais will discuss the multichannel FNS systems which are being developed at the Cleveland VA Medical Center and Case Western Reserve University in Cleveland, $\mathrm{OH}$, USA. The only FNS system to reach the stage of clinical trials, the Parastep ${ }^{\circledR}$ System (Sigmedics, Inc, Northbrook, IL, USA) will also be described briefly. Finally, a representative neuroprosthetic user, Dr Ronald D Lew, and a representative neuroprosthetic prescriber, Dr Kristjan T Ragnarsson, will respond to the presentations.

\section{LSU-RGO with muscle stimulation (Dr M Solomonow)}

The Louisiana State University reciprocating gait orthosis (LSU-RGO) powered with electrical muscle stimulation was designed to provide the biomechanical functions necessary for the simplest form of locomotion: standing up, standing and balancing, and simultaneous swing-phase and 
contralateral push-off of the legs. ${ }^{2-4}$ The LSU-RGO is a passive mechanical orthosis generally categorized as an HKAFO (hip, knee, ankle, foot orthosis). ${ }^{5,6}$ It uses a specially designed ratchet knee joint which can lock the knees at less than full extension. The hip joints are connected to each other with a metal or plastic pelvic band and with two stainless steel cables inside a low-friction conduit. The cables prevent simultaneous flexion of both hips and conse- quent collapse, and they provide force transmission from one hip to the contralateral one to make reciprocal movement of the legs possible. The reciprocal mechanism may be disengaged to allow simultaneous flexion of both hips for sitting down.

The RGO (Fig 1) allows stable, upright balance at minimal metabolic energy cost. Muscle stimulation in the LSU-RGO system provides the power for locomotion while releasing the upper extremities and trunk (a)

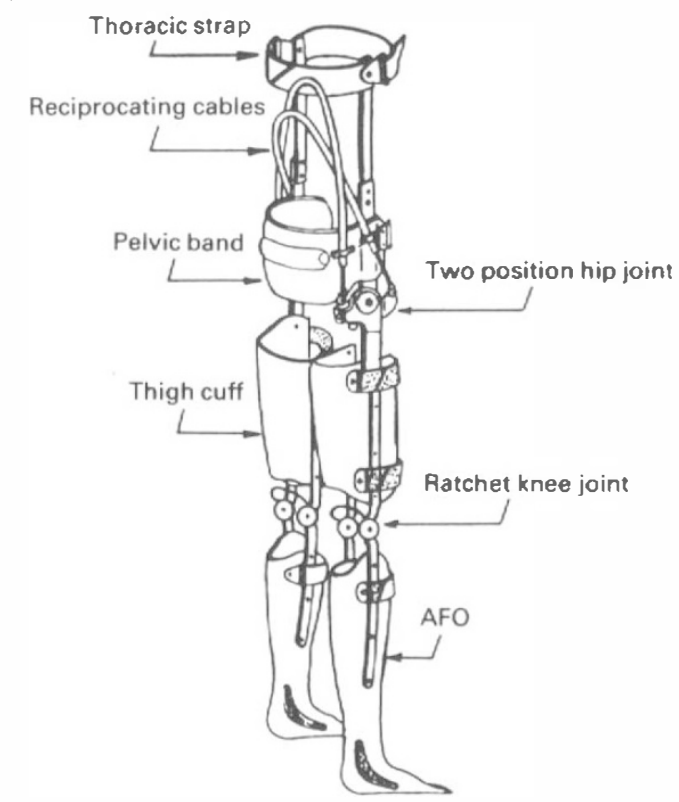

(c)

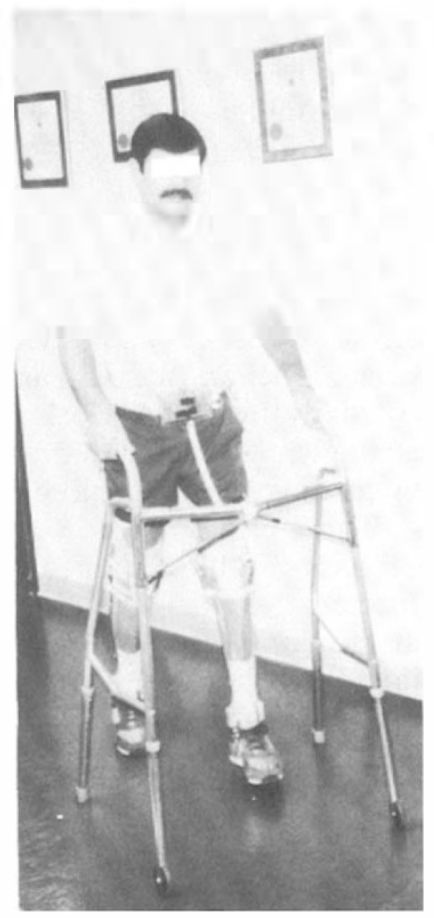

(b)

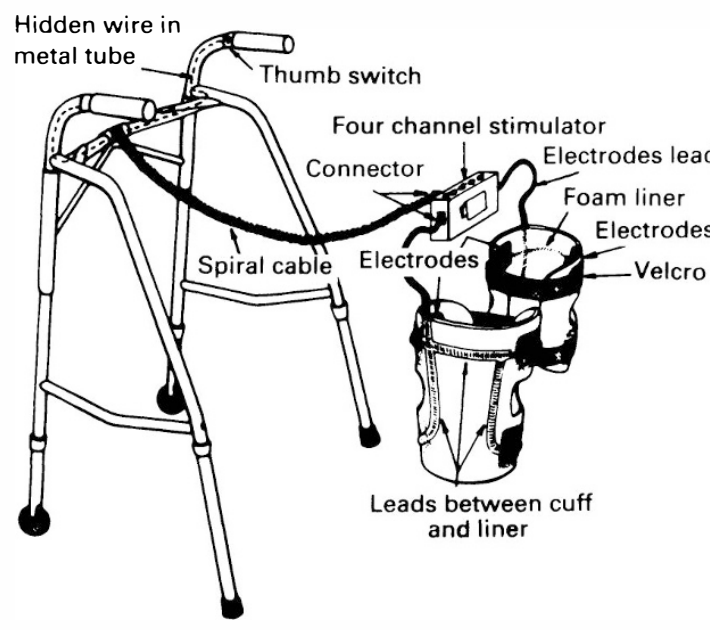

Figure 1 (a) A schematic drawing of the LSURGO. (b) A schematic drawing of the stimulation system designed for use with the LSURGO by paraplegic patients. (c) A person with paraplegia at the $\mathrm{T} 7$ level ambulating with the LSU-RGO powered by the electrical stimulation system. 
muscles from bearing all of the burden of that task. Adding FNS reduces the energy expenditure per unit of body mass ${ }^{7-9}$ because both the upper and lower extremities are active during locomotion. Two pairs of stimulation channels are used with both members of a pair active simultaneously. The stimulation is accomplished with monophasic, charge-balanced pulses of $0.5 \mathrm{~ms}$ duration at a rate that varies from 18 to $26 \mathrm{pps}$, according to the individual patient. The rate adjustment allows contraction of a strength near $50-60 \%$ of the maximal tetanic force without inducing fatigue. Individual adjustments can be made to accommodate the muscle fiber composition and degree of atrophy in each patient. Stimulation interface to the muscle is accomplished with carbon-impregnated rubber electrodes covered with Karaya ${ }^{\circledR}$ solid gel (Unipatch, Inc, Wabash, MI, USA). Electrodes and wires are integrated into a flexible copolymer electrode cuff. Control switches are mounted on each handlebar of the rolling walker.

At present, over 5000 units of the RGO have been custom fitted to patients all over the world. The RGO powered by electrical stimulation has been fitted and evaluated on a more limited group of appoximately 45 patients at the LSU bioengineering laboratory, and at collaborating centers in France, England, Italy, The Netherlands, and South Africs. The levels of patients' neurological lesions range from $\mathrm{T} 1$ to $\mathrm{T} 10$. The LSURGO with FNS is fitted to the patients on a custom-made basis which is exempt from the requirement of endorsement by the US Food and Drug Administration (FDA). The results of the last 5 years of clinical work on the system position us to seek premarket approval from FDA in 1993.

Recent experience convinces us that a wide range of patients are candidates for this walking system including people up to $60+$ years old; people with complete and incomplete thoracic, and some low cervical injuries, and as long as 22 years post injury; patients with moderate to pronounced spasms and contractures; and overweight individuals. One must recognize, however, that the amount of training required to bring a 'complicated' patient to a satisfactory level of locomotion may be as much as five times that for an ideal patient.

Medical screening checks the stability of the spine, the integrity of the lower extremity joints, the condition of the long bones, and the soundness of the cardiovascularpulmonary system. Pressure sores in key locations and drug abuse are contraindications. Furthermore, the effect of electrical stimulation on pregnancy, pacemakers, and hypertension is not known and such patients are at present excluded. High motivation is an absolute requirement and it may compensate for some physical deficiencies such as spasticity and overweight.

The cost of the system including hardware and training averages $\$ 12,000$; higher for complicated cases and lower for simple cases. The cost of the RGO and associated therapy such as gait training is reimbursable by commercial insurances and Medicare/ Medicaid. In general, insurance carriers need to be better informed about neuroprosthetic devices and include them in their approved coverage.

The mechanical and electrical components of the system are custom made and fitted for each patient. Three stages of training, each 6 weeks long (for the average candidate and longer if there are complications), prepare the paralyzed person to use the neuroprosthetic system: muscle stimulation therapy, gait training with the RGO and, finally, gait training with the muscle stimulation-powered RGO. Following the training period, patients use the system for home, work, and recreational activities at varying levels depending on their own physical condition, skill, and desire.

Problems associated with manufacture of the RGO are minimal, and limited primarily to training orthotists to properly fabricate and fit the orthosis; such training is available in the LSU orthotics section which has been engaged in this activity for over 17 years. The various components are available directly from the manufacturer, DurrFillauer Orthopaedics Inc, Chattanooga, Tennessee, USA. The electrostimulation equipment is currently manufactured in limited quantities in the LSU bioengineering laboratory. Efforts are underway to form a manufacturing facility and to obtain 
FDA approval for commercial distribution. The RGO has become highly reliable over the years; in daily use, it requires about two service visits per year.

We evaluated the muscle stimulationpowered RGO for its utility in several types of occupational environment and in several activities of daily living. In the workplace environment, a paraplegic subject using the system could engage in activities such as operating milling and lathe machines, and packing small to moderate sized boxes; and office tasks such as filing documents and making phone calls, all while standing up and using both arms. Limitations were confined to the ability to exert large forces such as those needed when lifting a large heavy box, carrying packages larger than $12^{\prime \prime} \times 12^{\prime \prime} \times 12^{\prime \prime}$, reaching below waist level, or picking objects up from the floor. Activities of daily living included cooking a meal and preparing a drink in a standard kitchen, barbecuing hamburgers outdoors, inspecting automobile engine fluids, dressing, and washing. Social and recreational activities included playing catch, attending a cocktail reception, and target shooting in the field. While there are still some limitations, selected paraplegic subjects could perform all these tasks satisfactorily. Maneuvering in the kitchen with the RGO and walker was easier than with a wheelchair and did not require expensive architectural modifications.

\section{Modular hybrid FNS orthosis (Dr B J Andrews)}

The concept of combining FNS with external bracing and motors mounted on the orthosis was proposed in 1972 by Tomovic et $a l^{10}$ and clinical applications were independently pioneered in Yugoslavia, Scotland, and the USA. Important advantages have since been demonstrated for hybrid systems over FNS or bracing used separately. They include delayed onset of FNS-induced muscle fatigue; improved stability of the pelvis and trunk for individuals with higher spinal lesions (C8-T5); simplified motion control by reducing the number of degrees of freedom; compensation for insufficient muscle force due to dennervation or reflex habituation; improved level ground walking; and in some systems (such as the LSU-RGO hybrid described above), a brace backup that is useful in the event of an FNS system failure. As with all orthoses, patient acceptance depends on the individual's assessment of the balance between benefit in terms of function, independence, and therapy and cost in terms of physical effort, reliability, encumberance, and cosmesis.

In recent studies of the LSU-RGO and the ParaWalker hip-guidance orthosis (HGO) developed at Oswestry, Shropshire, UK, both with no FNS, adults often discontinued using the braces because of encumbrance, muscle fatigue, and interference with activities of daily living such as toileting. ${ }^{11}$ The present designs of RGO and $\mathrm{HGO}$ are not convenient for transport or to get into and to get out of, from a wheelchair. Adults use them primarily during exercise therapy workouts. If these devices are to augment the use of a wheelchair for spontaneous locomotion in the home and community, further reductions in upper limb physical effort and greater convenience are required.

The modular hybrid FNS orthosis was developed to increase function in an RGO by adding free knee flexion during swing and better control of hip and ankle motion. The modular HKAFO system has two parts; the first, an anterior floor reaction type AFO, is worn continuously on the affected $\operatorname{leg}(\mathrm{s})$ to provide assistance with transfers, stand-sit maneuvers, and limited range ambulation; the second part, a hip/trunk brace component, is conveniently put on or taken off (under or over the clothing) as required to improve ambulatory function or reduce upper limb involvement. This twopart arrangement should circumvent the interference to activities of daily living associated with the thoracic brace section of the RGO.

The anterior floor reaction orthosis (FRO $)^{12-14}$ works on the principle of allowing the knee to be conditionally stable in extension without muscular action by directing the ground reaction force anterior to the anatomical knee joint axis. The FRO has now been used in paraplegic subjects with no preserved voluntary control of knee 
extension by applying stimulation to the quadriceps only when the ground force vector passed through or behind the knee axis. ${ }^{15}$ In this way, the leg was stabilized and knee buckling was prevented. Because the force vector is mainly oriented ahead of the knee joint during standing and the stance phase of walking, the duty cycle of activation of the extensors is reduced to a few

(a)

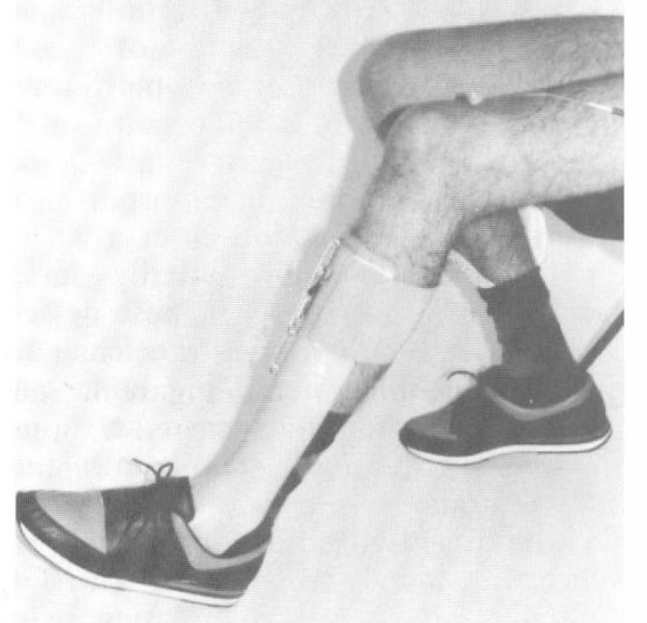

(c)

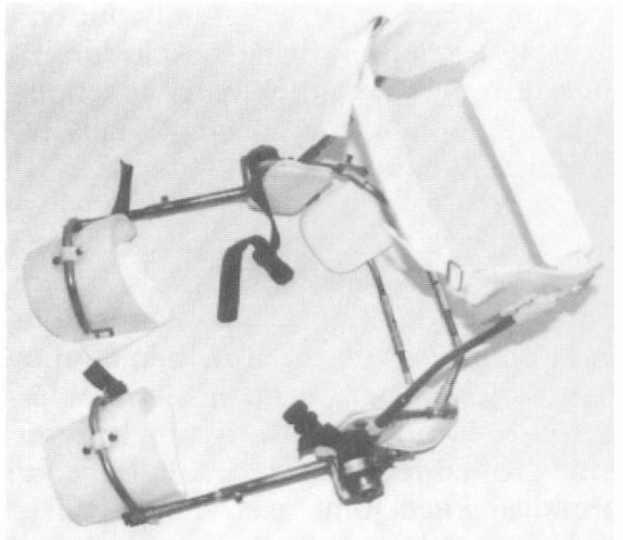

percent, thus avoiding FNS-induced muscle fatigue. ${ }^{16}$ For reasons of safety, the application of the FRO had previously been restricted to those patients who had sufficient preserved voluntary knee control and who had been adequately trained to avoid or recover from buckling incidents.

A new design of FRO developed for this application (Fig 2) features lightweight

(b)

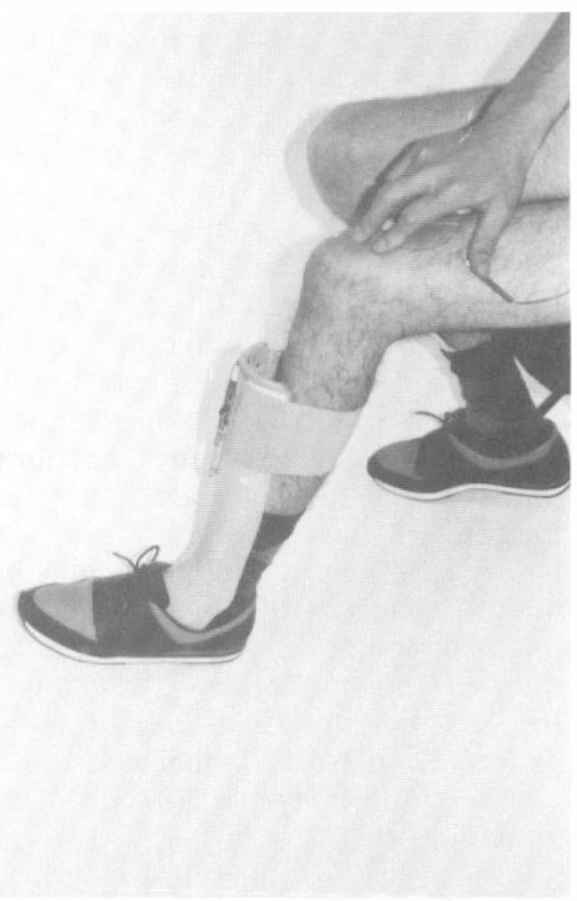

Figure 2 Prototype components of the 2-part modular hybrid FNS system. (a) and (b) show the anteriorly molded FRO with plantarflexion control provided by the elastic calf strap. (c) shows the lightweight, easily put on and taken off, hip/trunk brace fitted with hips joint controlled by a wrapped spring clutch. 
(typically less than $0.4 \mathrm{~kg}$ ), stiffer resistance to dorsiflexion than conventionally molded AFOs, and better control of plantarflexion. The FRO has been used by paraplegic individuals for FNS-assisted standing ${ }^{16}$ as well as for reciprocal ${ }^{17}$ and swing-through crutch-aided ambulation. Preliminary laboratory data are available for spinal cord injured persons using the FRO both with surface electrodes ${ }^{17.18}$ and with percutaneous electrodes. ${ }^{19.20}$ The gait modes are limited by the physical demands on the user's upper body musculature. ${ }^{21}$ Thus, in order to extend the distance traversed, the user must take rest breaks, preferably while standing, to avoid the need for a suitable seat. Speed of locomotion is slow, typically $0.1-0.2 \mathrm{~m} / \mathrm{s}$, and depends on the FNS control mode. Recent preliminary data from trials with surface FNS suggest that swingthrough gait involving bilateral flexion of the legs can be faster (typically $0.6 \mathrm{~m} / \mathrm{s}$ ) and no more energy consuming than simple FNS reciprocal gait. ${ }^{22}$ At present, the duration and frequency of rest periods that optimally extend the range are unknown.

The first hip/trunk brace module incorporated a reciprocating mechanical linkage of the hip joints, using a flexible linear bearing. ${ }^{23}$ The method and advantages of reciprocally linking the hip joints in an RGO using Bowden cables have been described in the discussion of the LSU-RGO system above and in earlier sources. ${ }^{5.24}$ The device can be worn over or under the clothing and a quick release locking mechanism is used to connect and disconnect the thigh cuffs to assist with putting on and taking off.

With the hip linking mechanism disconnected, the user stands up applying the same FNS control as with the FRO alone. Once upright, the user extends the hips until the LSU-RGO-type hip joint locks engage. A similar FNS controller to that developed for use with the FRO alone can be used to assist 4-point gait. ${ }^{18}$ In contrast to fixed knee walking, ambulation with the modular orthosis exhibits improved ground clearance and dynamic cosmesis which are less dependent on the rigidity of the hip/trunk brace.

Preliminary results are available on two midthoracic spinal cord injured (SCI) indi- viduals with complete lesions. Standing and walking were much more secure than with the FRO alone because bilateral hip flexion during the double support phases was prevented by the hip linking mechanism. The patients reported less need to use the upper limbs than with the FRO module alone. The hip/trunk brace could be put on and taken off in less than $20 \mathrm{~s}$ from the wheelchair; when not in use the brace could be folded and stowed on the wheelchair backrest or beneath the seat for ready access. These preliminary laboratory-based results indicated further development of the hip/trunk brace module. For example, the mechanical linkage did not allow the potentially faster swing-through gaits or work activities such as leaning forward to pick up objects from a low surface. If the linking mechanism is disconnected, then it provides no hip motion control. For this reason, a computer-controlled electromechanical hip joint was developed based on the wrapped spring clutch principle illustrated in Figure 3. Each hip joint may be rapidly switched between two states by the FNS control computer using the miniature servomotor. The servo moves the control tang of the drive spring to produce two states: 'free' or 'engaged'. The 'free' state allows free hip flexion and extension; in the 'engaged' state the joint acts like a silent toothless ratchet allowing hip extension but no flexion. Switching the states of the hip joints in phase with on-line detected gait events and FNS muscle activation allows enhanced control of locomotion with automatic mode changing.

Presently, the system is being used only in the laboratory with paraplegic subjects performing sit-stand maneuvers, standing with forward leaning, 4-point and 2-point reciprocal gaits, swing-to and swing-through gait. These combinations of FNS and advanced bracing incorporating sensors and active control elements herald the next generation of hybrid systems.

In evaluating the physical effort of using a modular hybrid orthosis, one must recognize the intermittent nature of neuroprosthesis use in the workplace. Metabolic energy expenditure is likely to depend on the skill of the user, on the pattern of intermittent usage, and on the condition of 
a

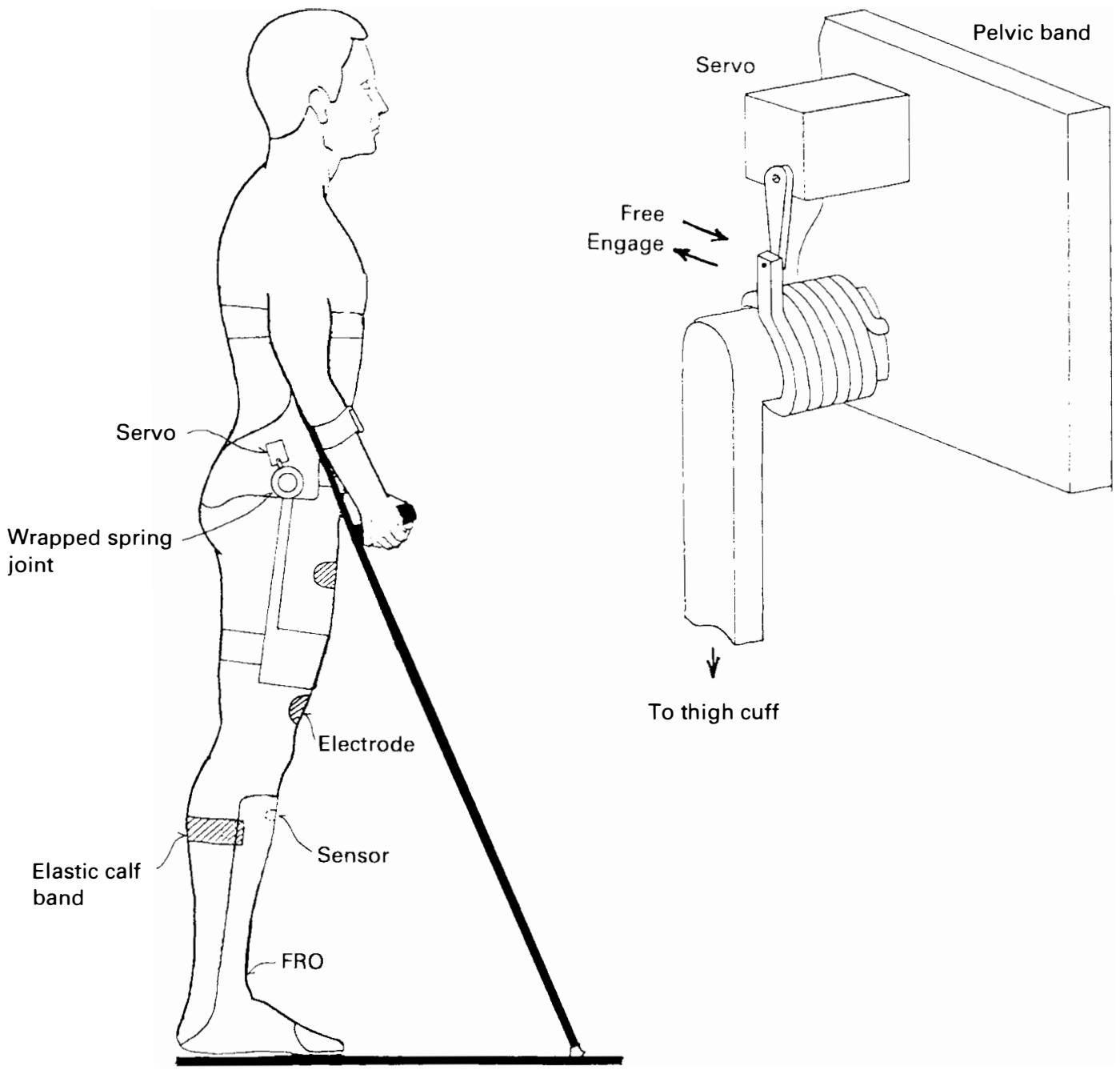

Figure 3 (a) Schematic diagram of the 2-part modular system. The wrapped spring clutch-controlled right hip joint is shown in (b). Here a miniature model servomotor is used to pull back on the control tang to unwrap the drive spring off the joint shaft friction hubs to allow the 'free' state unimpeded flexion/extension. In the 'engaged' state the drive spring will wrap down onto the hubs during flexion creating a strong resisting frictional torque. Extension will be unimpeded since it will tend to unwrap the spring.

the shoulder girdle musculature. The use of FNS will add to metabolic expenditure, but it is hoped that its use will reduce upper limb muscle forces particularly in those small muscles ill adapted to sustain them. Overall, with the simultaneous work of upper and lower body, it is hoped that less energy will be required.

After laboratory tests conducted locally and appropriate safety checks and certification are completed, trials in independent clinical centers will follow. Issues to be resolved include patient selection criteria, training of clinical and support staff to fit the systems, training protocols for neuroprosthetic system users, amount of technical support from the system manufacturer, and pattern of home and community use. 
At present, only the FRO and a surface electrode FNS controller are ready for independent clinical trials and technology transfer. This is planned to begin in 1992 in collaboration with a number of the Canadian clinical nodes in the network of centres of excellence 'Neural Regeneration and Functional Recovery', established in 1990 by the Medical Research Council of Canada.

\section{Cleveland VA-CWRU functional neuromuscular stimulation system (Dr E B Marsolais)}

Forty-eight channel FNS systems employing intramuscular electrodes are under development at the Cleveland VA Medical Center and Case Western Reserve University (CWRU). Those systems provide active FNS control of the spine, hip, knee, and ankle joints. Functional walking capabilities of paraplegic users include maximum distance of 1200 meters, maximum speed of $1.0 \mathrm{~m} / \mathrm{s}$, side and back stepping; an inter- mittent capability of energy utilization of less than $50 \%$ of maximum aerobic capacity; and steady walking at $0.6 \mathrm{~m} / \mathrm{s}$. These data are based on experience with more than 20 subjects with neurological lesion levels between T4 and T12 who used the systems for walking in the laboratory, for seated exercise at home, and for selected Institutional Review Board approved activities outside of the laboratory. While the 48-channel system represents a major functional capability, current technical limitations will probably delay its widespread application for about 10 years. This discussion will, therefore, be restricted to that VA-CWRU system which is close to multicenter clinical testing.

The currently available VA-CWRU lower-extremity stimulation system consists of an 8-channel permanently implanted stimulator with eight permanent intramuscular electrodes (Fig 4). ${ }^{25}$ The design has been used in upper extremity neuroprosthesis patients for over 5 years. The device is both powered and controlled by radiofrequency transmission from an external power

\section{(ELECTRODE)}
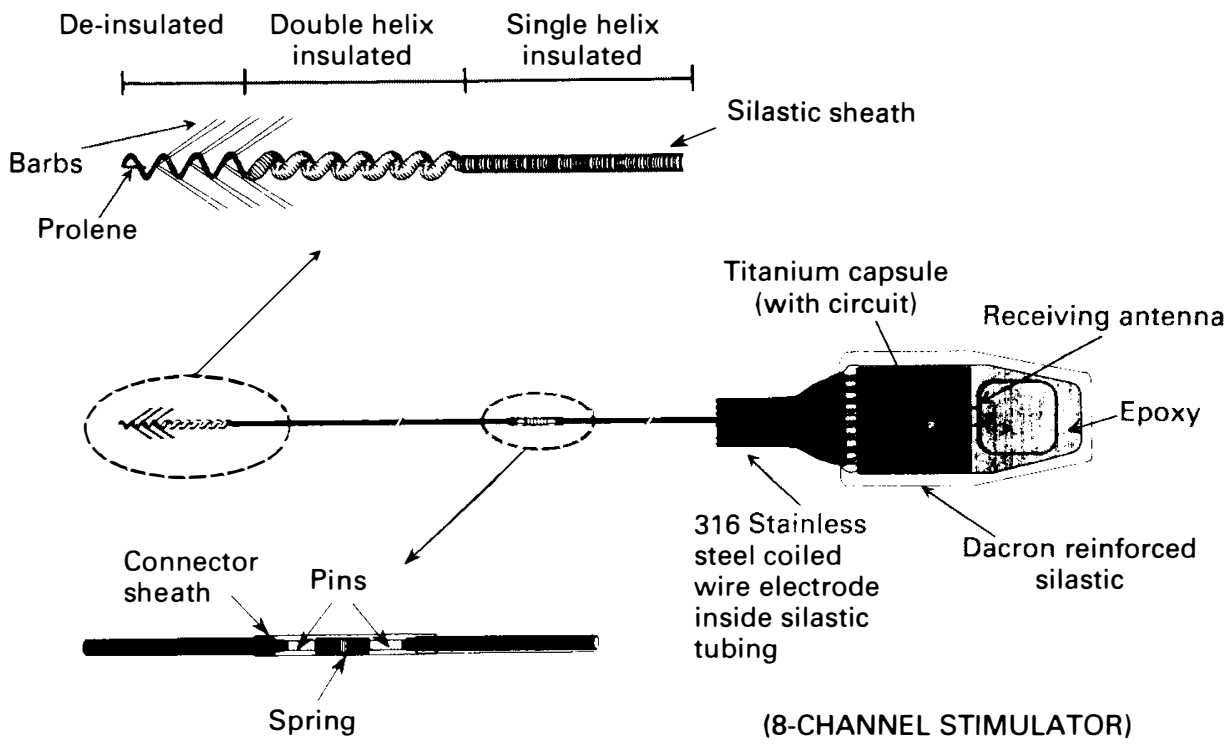

(INTER-CONNECTOR)

Figure 4 VA-CWRU 8-channel implant stimulator. An interconnector allows connection of the electrodes to either percutaneous leads or the implant stimulator. (Only one of the eight identical electrodes is pictured.) 
pack and controller. The device is implanted subcutaneously in the lower abdomen and the external antenna loop is held in place over the implant site with a hernia belt (Fig 5). The device can be used by paraplegic people for standing, by quadriplegic people for pivot transfer, or by people who have suffered a stroke or traumatic brain injury (TBI) for gait augmentation. Conventional bracing will not be required in most patients using the 8-channel system but in paraplegic individuals the system can be combined with bracing to allow walking.

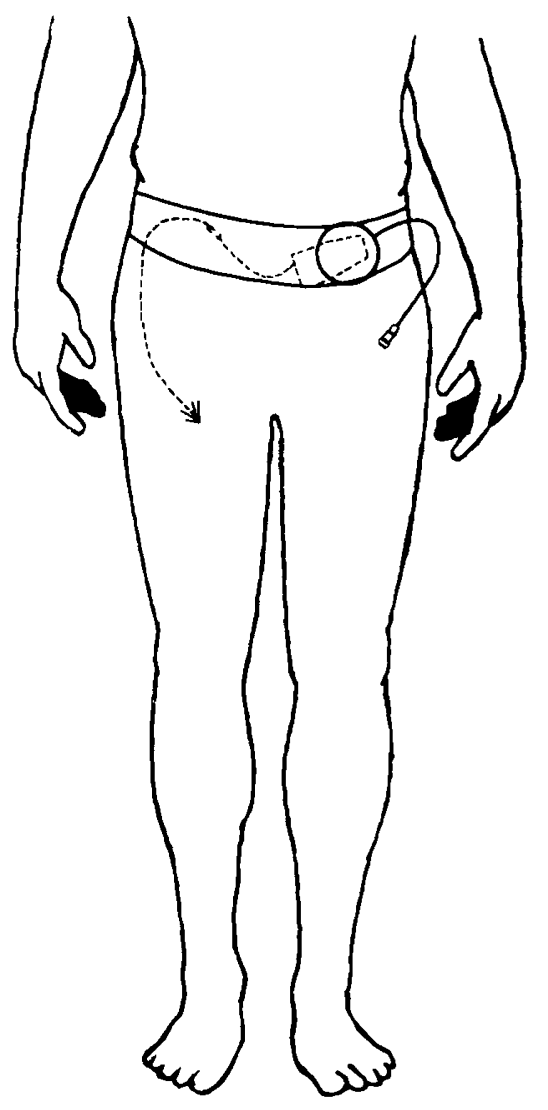

Figure 5 Internal and external parts of 8-channel VA-CWRU system. Shown with dotted lines are the stimulator and one of eight electrodes implanted in the body. To achieve the radio-frequency link, the antenna is held over the implanted stimulator by a hernia belt. A connecting cable links the antenna to the external controller. External components are shown with solid lines.
Inclusion criteria for device recipients are: a strong desire to have the implant; impaired lower extremity motor function due to an upper motor neuron lesion; an intact lower motor neuron system; hip and knee range within 5 degrees of neutral; ankle range within 2 degrees of neutral; and a high 'gadget tolerance.' The device recipient must have sufficient residual motor function to manipulate the necessary equipment and a triceps on at least one side of $4+$ strength (able to resist force additional to gravitational forces). ${ }^{26}$ Contraindications include transient ischemic attacks, general debilitation, personality disorders, active drug abuse, passive personality, skin problems, and a low pain tolerance.

Surgical implantation is done in two phases by a specially trained spinal or neurological surgeon. In the first phase, electrodes are implanted with an outpatient, nonincisional technique which employs modified hypodermic needles; and which leaves temporary electrode leads outside the body where they can be connected to an external stimulator for muscle training. In the second phase, when the stimulator is implanted, the temporary percutaneous leads are removed and the electrodes are connected to the implanted stimulator, also in an outpatient procedure. The techniques can be mastered by surgeons who attend a short training course; a surgical technique manual is being written to aid in this technology transfer. For those patients with sensation, a local anaesthetic augmented by an oral analgesic is used. The electrodes are allowed to stabilize for 6 weeks prior to implantation of the stimulator. That allows both the treatment team and the patient a chance to evaluate the results of the system prior to committing to the stimulator implant.

Investigational trials of the FNS 8-channel system are currently underway in incomplete quadriplegic individuals. While we have had considerable experience in dealing with implanted stroke patients, ${ }^{27}$ we are just developing the protocols for lower extremity training in quadriplegic individuals. We are utilizing standard therapy techniques as much as possible and are monitoring function closely. We have implanted one 
complete system in a partial quadriplegic individual (Frankel C, C6-7) and a second similar patient has just started the program. There appear to be no major safety problems. The level of functional gain is significant - the individual can transfer without assistance using the FNS system. He uses the system at home for exercise and for those transfers that he is unable to do with residual voluntary muscles. The device is easy to use with minimal putting on and taking off times; the user interface was customized for this subject based on his degree of hand dexterity and cognitive needs. The system does not detract from the individual's appearance and requires only moderate metabolic energy levels for function. Modification of the workplace should not be needed over that currently legally mandated.

The implant stimulator was manufactured, under contract, by Biocontrol Technology Inc, Indiana, Pennsylvania, USA, based on the original CWRU design. The electrodes were manufactured in the VACWRU laboratories. As there has been only 18 months of experience with the permanent intramuscular electrode, more time is needed to assess its long term stability. Further miniaturization of the external portions of the system is needed to facilitate user acceptance. Improved foot contact sensors will be important for some uses.

Potential problems for use of the implanted FNS system include infection, nerve injury, burns, bone fractures due to falls, and possible interference of this device with other implanted electronic devices. There has been considerable concern about the potential development of Charcot joints. While it is certainly possible to develop degenerative joint changes due to abnormal use of insensate joints, true Charcot joints develop in joints which lack a sensory connection to the spinal cord as in diabetic neuropathy. In upper motor neuron lesions, this sensory connection remains intact even though the individual is unable to consciously appreciate the sensation. The other protecting factor is that the duty cycle for all currently proposed applications is much less than that to which the body is normally subjected, leaving a significant margin of safety. Should a joint become damaged to the point of not being usable, then bracing or joint reconstruction would be rehabilitation alternatives.

About 3 months of one hour of stimulation per day are needed to develop fatigue resistance and aerobic capability in the stimulated muscles. In rehabilitation of the head injured and stroke population, the FNS treatment regimen mixes well with our conventional therapeutic regimens. Systems are available in quantity. Maintenance manuals will cover hardware troubleshooting and surgical techniques for stimulator and electrode replacement should these prove necessary.

Quadriplegic individuals using the stimulation system for pivot transfer lack finger function and need a special stimulator control design (Fig 6). ${ }^{28}$ The current quadriplegic muscle implant protocol includes quadriceps, hamstrings, posterior adductors, and erector spinae muscles bilaterally (Fig 7). This will allow selected complete quadriplegic individuals assisted transfers, automatic sitting pressure relief, and aerobic exercise. Some people with incomplete quadriplegia will be able to do independent transfers using this system. Paraplegic individuals with arm impairment will be able to do independent transfers with less arm and

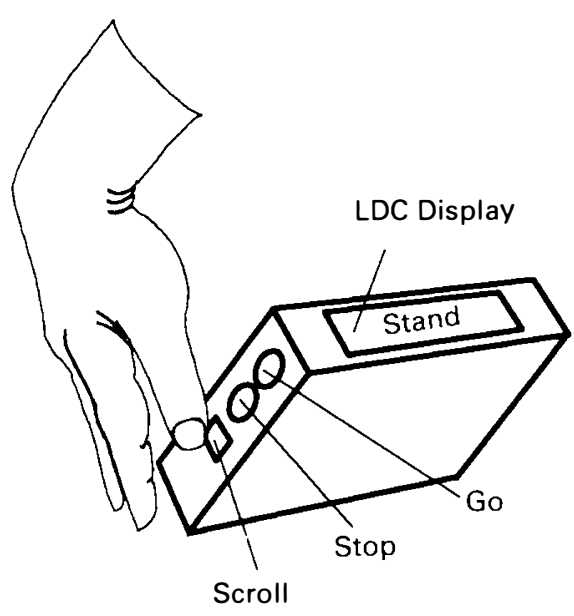

Figure 6 Control interface for VA-CWRU 8-channel system for quadriplegic user. The interface connects to the cable from the antenna (Fig 5) and is worn on the user's belt. 


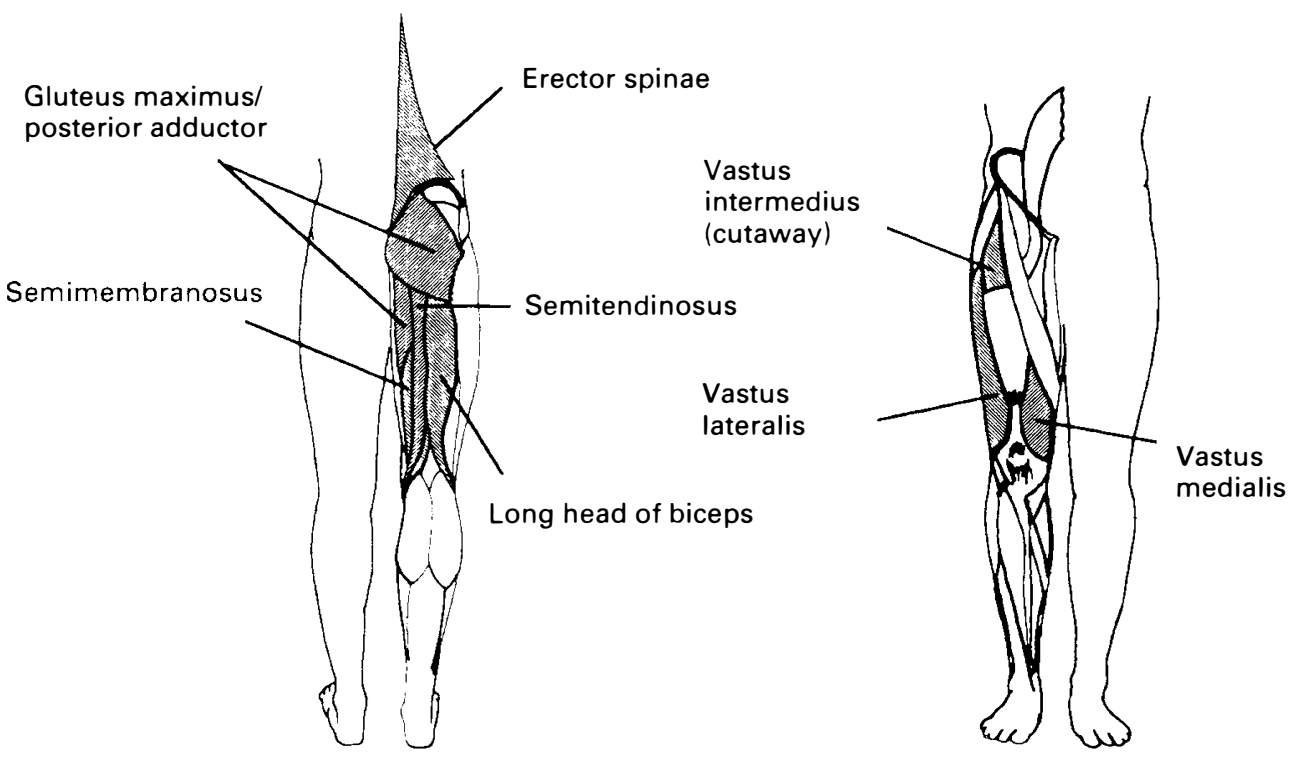

Figure 7 Muscles instrumented with electrodes in the VA-CWRU quadriplegic 8-channel system.

shoulder use. Selected paraplegic individuals may desire to use the hip and spine control capabilities of the system in conjuction with bracing to allow active walking including stair climbing. Due to the severe channel limitation, such a system would remain energy inefficient and quite slow, but would provide the advantages of standing stability from the conventional bracing and stair climbing from the FNS.

The 8-channel system with percutaneous leads and an external stimulator has been evaluated also in stroke and head injured individuals. These users are equipped with a simple on-off control for walking function determined by heel and toe contact switches. The severely involved stroke individual is able to stand and usually to walk without bracing using the 8-channel FNS system. ${ }^{29}$ Preliminary data indicate that, in addition to correcting gait defects, use of the FNS system together with gait training may help some patients to regain voluntary muscle function. Electrodes are deactivated as the voluntary control is regained.

Further clinical trials are needed to effectively extend FNS application. We currently have an FDA investigational device exemption (IDE) to use the 8-channel implant in our center 'for ambulatory function for those who are paralyzed'. ${ }^{30}$ This includes stroke, head injured, paraplegic, and quadriplegic individuals. Investigational trials in each diagnostic group will take place when designated funding is secured. A commercial partner will be needed for production and eventual marketing of the device. When investigational trials have been successfully completed, application will be made to the FDA to conduct multicenter clinical trials. They will require participation of approximately 50 patients and the development of a system of interactive referring centers. We hope to begin such trials in one year. The last step towards workplace use of the VA-CWRU system will be to obtain premarket approval from FDA.

\section{The Parastep ${ }^{\circledR}$ system (R A Habasevich PT)}

The Parastep ${ }^{\circledR}$ system uses a 6-channel, microprocessor-controlled stimulator and surface electrodes to produce standing up and stepping as described by $\mathrm{Kralj}^{31}$ and Graupe. ${ }^{32}$ The device stimulates the quadriceps and hip muscles to extend the knees and hips to achieve standing. Peroneal 
nerve stimulation produces a flexion withdrawal reflex to shorten the limb in preparation for stepping. The microprocessor then cycles on quadriceps and hip activation to complete the step. The user positions his body over the advanced leg with use of an adapted walker and then repeats the process on the other side to achieve reciprocal gait. The stimulator is worn at the waist and the control switches are on the walker; no bracing is used in the current version. The functional goal is upright mobility-including moving through doorways and up a one-step curb-as an alternative, not a substitute, for the wheelchair.

FDA-approved clinical trials have been underway since late 1990 at 20 sites with over 100 patients. They are mostly SCI patients with complete lesions at levels from $\mathrm{T} 3$ to $\mathrm{T} 12 ; 75 \%$ are male, mean age is 32 years with a range of 18-52 years. Each patient participates in the study for 4 months which cover 32 physical therapy sessions. At the conclusion of the trial, a patient who demonstrates safe and effective use will have the option to purchase the motor prosthesis at cost-between $\$ 7,000$ and $\$ 10,000$.

Results from over 2000 hours of trials show that users all experience a high metabolic energy cost which varies with proficiency in their use of the system. Energy use is high because all propulsion is through the shoulder girdle or arms. Mastery in using the device depends on the disability level, variations in skill acquisition, and frequency of use.

The Parastep ${ }^{\circledR}$ system is technologically simple compared with some of the other systems which have been described here. However, it is the first ambulation system to undergo clinical testing in an approved multicenter study. A successful outcome should establish a precedent for FDA approval of FNS devices for functional restoration. This is important because current FDA labelling requirements restrict FES/FNS to the following uses: retarding or reversing atrophy, increasing muscle blood flow, muscle reeducation, maintaining or increasing range of motion, inhibiting spasticity, and preventing venous thrombosis in postsurgical patients.

\section{Comments of a potential neuroprosthetic system user (Dr R D Lew):}

I am an employed paraplegic who is a daily user of a 48-channel investigational FNS system. I use the system for exercise according to an FDA-approved research protocol.

During the symposium, no one addressed the impact on potential FNS users of the newly passed Americans with Disabilities Act (ADA). It appears to me that compliance with the ADA would remove most, if not all, of the barriers for people with disabilities to get into the workplace. The discussions of the various neuroprostheses should therefore be limited to functioning within the workplace, be it office or home. Only Dr Solomonow spoke about the utility of his system in the workplace. Developers of neuroprostheses appear to be focused on locomotion rather than on functional standing. Locomotion is important in the workplace no matter how short the distance. Yet, if an orthosis can only provide very slow locomotion, it will rarely be chosen over the wheelchair which is faster, safer, and more energy efficient.

Dr Solomonow gave an excellent description of the LSU-RGO prosthesis and he explained the function of his system in the workplace. He noted the delicate balance that is needed with his system, the lack of bending at the waist, and the inability to climb up and down stairs. While he showed how his orthosis could be adapted to the workplace, he did not elaborate on how it could be made more functional in the future. He did not discuss the extent to which low bone mineral content in the leg bones or poor cardiovascular fitness would disqualify an individual from using his orthosis.

The main reasons for current orthotic systems being discarded are high energy cost per meter traveled, excessive patient encumbrance, and lack of cosmesis. Dr Andrews' solution is a modular hybrid system comprising an anterior floor reaction orthosis (FRO) and a hip/trunk brace that can be worn as required for enhanced function. Besides the apparent easy putting on and taking off of the hip/trunk brace, this hybrid system is similar to the LSU-RGO. 
Yet, the energy costs for ambulation seem high. Although no figures were given, the energy costs may approach those seen in ambulation with FNS alone. Unfortunately, Dr Andrews' presentation focused on locomotion to the workplace; he did not discuss how to make the lower extremity motor prosthesis user functional in the workplace. I have several questions concerning standing with his system. Can the user of his system stand with both hands free? How much of a perturbation can he sustain before being knocked off balance? How much additional support will he need to achieve functional standing?

Dr Marsolais spoke primarily about an 8-channel FNS system. He noted that it could be used by paraplegics, quadriplegics, stroke victims, and brain-injured individuals. Since the symposium's focus is on occupational environments, the presenters should concentrate on those most likely to be employed who would benefit from lower extremity prostheses-paraplegics. Most survivors of TBI have cognitive and behavioral deficits that would make them less than ideal candidates for many occupations.

The obvious advantages of Dr Marsolais' FNS system are the easy putting on and taking off, and the cosmetic appearance. The obvious disadvantage is the high energy usage. Dr Marsolais did not describe how his 8-channel, or even his 48-channel, system functions in the workplace. The 8channel system that was described would allow a paraplegic person to stand but he could not ambulate because he does not have the necessary innervated musculature. The concern that I have is: can the 8channel system user stand with the same stability as the LSU-RGO or hybrid modular user? From my experience with tests of only eight channels of my 48-channel system, the answer is no. Movement in a confined space would require the use of the upper body musculature. Any activity with the 8-channel FNS system has a higher energy cost than with the two hybrid systems. If bracing is added to the 8-channel system for selected individuals, the major advantages will be negated. It is my impression that the 8-channel system has little benefit for the lower extremity neuroprosthetic user in the workplace.

I do not use the VA-CWRU FNS system in my occupation. I find that a standing wheelchair allows me the freedom to perform my job with the least amount of inconvenience. I do not have the stability that I would like while standing, but I can bend and do some one-handed tasks. I would not choose Dr Solomonow's LSURGO with muscle stimulation because of the slow locomotion, the encumbrance of the orthosis, the instability of the orthosis in the standing position and the inability to bend at the waist. Besides the encumbrance of the system, I would not choose Dr Andrews' system for the same reasons. Its energy requirement appears to be significantly higher than that of the LSU-RGO. I don't use Dr Marsolais' 48-channel system in my work because it does not provide enough standing stability; it does not offer any advantages over a standing wheelchair in my daily occupational duties.

The Parastep ${ }^{\circledR}$ system was shown at the conference and I feel it offers less stability for standing than any of the other systems. While the locomotion it provides is adequate, it offers little for the lower extremity user in the occupational work environment.

\section{Comments of a potential neuroprosthetic system prescriber (Dr K T Ragnarsson):}

During this symposium, the presenters outlined the general direction towards a clinically usable neuroprosthetic system and reported that progress has been slow. Thirty years is a long time to develop a new clinical intervention. Although cardiac pacemakers may be less complex FNS systems than motor prostheses, it is of interest that only 40 years ago the first reports were published on the use of electricity to correct abnormal heart beat with an external stimulator and electrodes. For several years thereafter, cardiac pacemaker systems were partly external while being widely tested, further developed, and prescribed clinically. Hundreds of cardiac pacemaker systems are now being implanted each day, but still the final prototype may not yet have been 
developed. The lesson to us is that we have to use what is safe, effective, and available to help persons with disabilities while at the same time trying to improve the devices.

Clinicians who care for persons with neurological impairment, including SCI, have been watching developments in the field of FNS with great interest for more than a decade and have been anxiously waiting to obtain something clinically useful from this research. So far, the only device for lower extremity FNS that has become available to clinicians is the FNS bicycle ergometer (ERGYS, Therapeutics Technologies Inc, Tampa, Florida, USA) which has been on the market for at least 6 years. It does not seem excessive to ask the investigators and developers to provide a neuroprosthetic device at a reasonable cost which is safe, and allows just some persons with SCI to stand up from a wheelchair with relative ease, stand with minimal arm support, ambulate for short distances on a level surface and perform active exercises of the lower limbs.

At this symposium excellent descriptions of different FNS systems for standing and ambulation have been given, but the focus questions have not been fully addressed. Several tasks can be identified in order to get 100 users of lower extremity neuroprostheses into the workplace.

\section{Determine the user population}

The current criteria used to select the ideal candidate for FNS ambulation include: (1) T5-T12 paraplegia with preserved lower motor neurons, (2) impaired sensation, (3) minimal or moderate spasticity, (4) joint range of motion within functional limits, (5) good health, and (6) psychological appropriateness (realistic, well motivated).

Adhering strictly to these criteria, it appears that a relatively small proportion of persons with SCI could be fitted with FNS systems for ambulation. Eliminating those with cervical and high thoracic cord lesions who would be poor candidates due to their physiologically reduced cardiovascular capacity, as well as those with lumbar lesions and therefore damaged lower motor neurons, no more than $20 \%$ of the entire
SCI population would fit the basic criteria. This number may be further reduced by as much as half when various exclusion criteria are applied: lower motor neuron lesion, age, obesity, joint contractures, excessive spasticity, medical problems, poor response to electrical stimulation, and inadequate motivation. That leaves, perhaps, less than $10 \%$ of the SCI population. Persons with incomplete SCI differ in their preservation of physiological functions and many of them, even some with cervical or high thoracic lesions, may be excellent candidates for FNS-assisted ambulation if their sensation is impaired to the extent that they can tolerate the electrical stimuli and if the incompleteness of the cord lesion preserves a degree of autonomic control.

It has been suggested that persons with stroke and TBI may benefit from FNSassisted ambulation, and this is undoubtably true in some instances. However, relatively few of the large number of persons who have suffered stroke or TBI will benefit from this technology. For example, motor deficits due to stroke can usually be adequately managed with a simple orthosis and most survivors of TBI have cognitive and behavioral deficits, but only rarely do they have motor deficits that interfere with ambulation.

While many investigators and developers of FNS devices suggest a very large potential user population for neuroprosthetic devices by including all individuals with SCI, stroke and TBI as potential candidates, it is not necessary to inflate this number unrealistically. From the clinician's point of view, it would be satisfactory to be able to prescribe a reliable and safe neuroprosthetic system for standing, limited ambulation or physical exercise, for only some persons with physical disabilities. As technology advances, and the understanding of human physiology improves, new techniques will become available to enlarge the group of potential users.

\section{Design the most appropriate FNS system}

The computer hardware and software for FNS systems already exist, but there is a considerable ongoing debate regarding the 
use of lower limb orthoses in conjuction with FNS systems. It is felt by many that FNS systems and orthoses used together are better in terms of safety and energy efficiency than either of the technologies applied alone. The orthoses used may vary from simple AFOs which only provide ankle control, to maximum bracing with HKAFOs, usually of the RGO design. Many investigators, including at least two of four presenting at this symposium, seem to advocate the maximum bracing for persons with thoracic paraplegia who use the FNS system for ambulation-HKAFO with an RGO design.

Few people with thoracic paraplegia use any prescribed lower limb orthoses for functional ambulation. At most, such orthoses are used for passive standing or exercise. Inconvenience and poor cosmesis may be reasons for discontinuing orthosis use, but these are only relative reasons. The key issues are the high energy consumption per meter traveled and the slow speed of gait. If persons with paraplegia were able to ambulate at a reasonable speed and at a low energy consumption level, most would accept the inconvenience of lengthy putting on and taking off time, and the poor appearance of most orthoses.

\section{Develop protocols for application, including implantation, programming, training and evaluation}

The subjects must be provided with the safest and most effective lower limb FNS system which is now available. It does not have to be the most sophisticated future prototype in order to be field tested or to be clinically prescribed. It is in order to clinically test the currently available 6- or 8channel systems with surface or percutaneous electrodes, which only allow standing up, weight shifting, exercising and ambulation for relatively short distances. Other generations of FNS systems are certain to follow, hopefully much improved, after various clinical and technical problems have been identified and solved.

If implanted devices are to be used, problems may be encountered. Can 100 subjects be found who will consent to surgical implantation of epimysial or nerve cuff electrodes, or perhaps of an entire FNS system? How will surgeons be trained in the different centers to perform these procedures? Is the technology safe? Is it effective? The limited experience with total implantation of upper extremity neuroprostheses may be helpful in this regard, although there are marked differences between the user populations and their goals.

It is most important that all successes and shortcomings of the tested FNS system be carefully documented in a multicenter clinical study as opposed to a single center investigation.

Necessary federal approvals must be sought and commercial firms must be identified for the production and marketing of lower limb neuroprosthesis. Since the market is relatively small and the potential clinical benefits great, investigators and clinicians must work harmoniously with commercial firms in order to see the endeavor succeed. In particular, my plea goes out to all the investigators in this field to work together and share their knowledge and technical resources. Many severely disabled people are waiting for FNS technology to help them. Facing their plight there is no room for large egos, individualistic ways of thinking, and personal hopes for the exclusive credit for having made this entire technology work. If it is going to work, it will be by collaborative effort. This collaborative effort must start immediately, if funding for research and further development is to continue, if commercial manufacturers are to be secured and, most important, if the population of persons with disabilities is to be justly served.

\section{Comments of the writer (C Kantor):}

While each of the neuroprosthetic systems presented needs particular technical modifications to improve its function and acceptance, all the systems have the common drawback of not providing an adequate combination of trunk mobility and stability. Both the LSU-RGO and the modular hybrid orthosis restrict trunk mobility in order to provide stability while the VACWRU system allows full trunk mobility 
but does not provide enough stability. Modifications in muscle selection, stimulation programming, bracing design, and user training are required to increase the function of each system. ${ }^{33}$

Disagreement about whether or not a first generation neuroprosthetic device should have implanted components suggests that the 100 workplace devices will be of several kinds. That reflects the diversity of functional goals of the 100 users. Individuals will define their own goals for workplace function and these may be accomplished only with different systems for different individuals. The earliest marketed systems will reach only minimal requirements; systems will evolve with refinement of new technologies and clinical experience.

All the systems demonstrate high metabolic energy cost per distance traversed. But most functional activities in the workplace are transient so that energy demand is intermittent. Ambulation for long times and distances is not a typical workplace requirement. High energy cost is not likely to be an obstacle to workplace use of neuroprostheses.

Any FNS system that will reach the marketplace must be tested in appropriately designed clinical trials. That will require the recruitment and training of appropriate clinicians. Developing the necessary interest among investigators is a major educational task, which we hope will be furthered by this paper.

Clinical trials will require funding support which is difficult to generate on a site-by-site basis, but perhaps even harder to generate from a single source. The ideal single source is a corporate partner willing to sponsor clinical studies on the way to securing approval to market the device. This level of involvement brings with it issues of liability, insurance, regulatory compliance, and market development. The corporate partner is an absolute requirement for each device developer to bring his neuroprosthetic system to wide availability for daily independent use by paralyzed people.

\section{Acknowledgements}

Dr Andrews' work was supported by the Alberta Heritage Foundation for Medical Research and the Medical Research Council of Canada. Dr Solomonow's work was supported by the Louisiana State University Board of Regents. Dr Marsolais' work was supported by the US Department of Veterans Affairs Rehabilitation Research and Development Service. A grant from the Buckeye Chapter of the Paralyzed Veterans of America supported the symposium reported here and the preparation of this paper.

\section{References}

1 Stallard J, Major RE, Patrick JH (1989) A review of the fundamental design problems of providing ambulation for paraplegic patients. Paraplegia 27(1): 70-75.

2 Solomonow M. Baratta R, Hirokawa S, Rightor N, Walker W, Beaudette P et al (1989) The RGO generation II: muscle stimulation powered orthosis as a practical walking system for thoracic paraplegics. Orthopedics 12: 1309-1315.

3 Solomonow M, Baratta R, Harris M, D’Ambrosia R (1991) Current status of walking orthoses for thoracic paraplegics. Mediguide Orthopaed 10: 1-8.

4 Solomonow M (1992) Biomechanics and physiology of a practical FES powered walking orthosis for paraplegics. In: Stein R, Peckham PH, editors. Neural Prostheses: Replacing Motor Function After Disease or Disability. Oxford Press, London.

5 Douglas R, Larson PF, D’Ambrosia R, McCall RE (1983) LSU reciprocation gait orthosis. Orthopaedics 6: 834-838.

6 Durr-Fillauer Medical, Inc (1983) LSU Reciprocating Gait Orthosis; Applications Manual. Chattanooga, $\mathrm{TN}$.

7 Astrand P, Rodahl K (1977) Textbook of Work Physiology: Physiological Basis of Exercise. 2nd ed. McGraw-Hill, New York.

8 Marsolais EB, Edwards B (1988) Energy cost of walking and standing with functional neuromuscular stimulation and long leg braces. Arch Phys Med Rehabil 69: 243-249.

9 Hirokawa S, Grim M, Le T, Solomonow S, Baratta RV, Shoji H et al (1990) Energy consumption in paraplegic ambulation using the reciprocating gait orthosis and electric stimulation of the thigh muscles. Arch Phys Med Rehabil 71: 687-694. 
10 Tomovic R, Vukobratovic M, Vodovnik L (1972) Hybrid actuators for orthotic systems: hybrid assistive systems. Proceedings of the 4th International Symposium on External Control of Human Extremities. Dubrovnik, Yugoslavia. Aug 28-Sept 2, 1972: 75-87.

11 Whittle MW, Cochrane GM (1989) A comparative trial of the hip guidance orthosis and the reciprocating gait orthosis. Health Equipment information: 192 (July). Department of Health and Social Security, London.

12 Saltiel J (1969) A one piece laminated knee locking short leg brace Orthot Prosthet June: 68-72.

13 Lehneis HR (1972) New developments in lower limb orthotics through bioengineering. Arch Phys Med Rehabil 53(7): 303-308.

14 Yang GW, Chu DS, Anh JH, Lehneis HR, Conceivao RM (1986) Floor reaction orthoses: clinical experience. Orthot Prosthet 40(1): 33-37.

15 Andrews BJ, Baxendale RH (1986) A hybrid orthosis incorporating artificial reflexes for spinal damaged patients. J Physiol 38: 19.

16 Mayagoitia RM, Andrews BJ (1990) Stability during standing for 30 minutes in a hybrid FRO. In: Popovic $\mathrm{D}$, editors. Advances in External Control of Human Extremities X. Belgrade, Yugoslavia, NAUKA.

17 Andrews BJ, Barnett RW, Phillips GF, Kirkwood CA, Donaldson N, Rushton et al (1989) Rule-based control of a hybrid FES orthosis for assisting paraplegic locomotion. Automedica 11: 175-199.

18 Andrews BJ, Baxendale RH, Barnett RW, Phillips GF, Yamazaki T, Paul JP et al (1988) Hybrid FES orthosis incorporating closed loop control and sensory feedback. J Biomed Eng 10(2): 189-195.

19 Muccio P, Andrews BJ, Marsolais EB (1988) Electronic orthoses: technology, prototypes and practices. $J$ Prosthet Orthot 1(1): 3-17.

20 Houdayer T, Freedman W, Triolo R, Andrews B, Betz R (1992) Prolonged standing for children with paraplegia by means of hybrid orthosis: a case study. IEEE (EMBS) Oct 29-Nov 1, Paris, France: 1349-1351.

21 Cliquet A, Baxendale RH, Andrews BJ (1989) Paraplegic locomotion and its metabolic energy expenditure. In: Rose FC, Jones R, and Vrbová G, editors. Comprehensive Neurologic Rehabilitation, Vol 13. Neuromuscular Stimulation: Basic Concepts and Clinical Implications. Demos, New York: 139-146.

22 Granat MP, Heller BW, Andrews BJ (1991) Synthesis of FES-assisted crutch aided swing-through locomotion in paraplegia. Proceedings of the IEEE (EMBS) 13th Annual International Conference, Orlando, FL, USA: 883-884.

23 Andrews BJ (1990) A modular hybrid FES orthosis for paraplegia. Proceedings of the IEEE (EMBS) 12th Annual International Conference, Orlando, FL, USA, 12(5): 2256-2257.

24 Scrutton DR (1971) A reciprocating brace with polyplanar hip hinges used on spina bifida children. Physiotherapy 57: 61-66.

25 Smith B, Peckham PH, Keith MW, Roscoe DD (1977) An externally powered, multichannel, implantable stimulator for versatile control of paralyzed muscle. IEEE Trans Biomed Eng 34(7): 499-508.

26 Kendall FP (1983) Muscles, Testing and Function. 3rd ed. Williams \& Wilkins, Baltimore, MD.

27 Marsolais EB, Kobetic R, Barnicle K, Jacobs J (1990) FNS application for restoring function in stroke and head-injury patients. J Clin Eng 15(6): 489-496.

28 Marsolais EB (1992) FES standing and transfer system for paraplegia and quadriplegia. J Am Paraplegia Soc 15(2): 145. Abstract.

29 Jacobs J, Kobetic R, Barnicle K, Marsolais EB (1991) Improved voluntary gait post stroke following treatment with the multi-channel, intra-muscular, microprocessor based FNS system. Proceedings of the 13th Annual Conference of the Rehabilitation Engineering Society of North America (RESNA), Kansas City, MO, USA, 1991: 283-285.

30 Federal Office of Device Evaluation, Center for Devices and Radiological Health (1990) IDE\#G900108.

31 Bajd T, Andrews BJ, Kralj A, Katakis J (1985) Restoration of walking in patients with incomplete spinal cord injuries by use of surface electrical stimulation-preliminary results. Prosthet Orthot Internat 9(2): 109-111.

32 Graupe D (1989) EMG pattern analysis for patient-responsive control of FES in paraplegics for walker-supported walking. IEEE Trans Biomed Eng 36(7): 711-719.

33 Marsolais EBM, Mansour JM (in press) Stability of the hip and trunk in paraplegic electrically augmented gait. IEEE Transactions in Biomedical Engineering. 